\title{
Family Medicine Physicians' Perspectives Regarding Rural Behavioral Health Care: Informing Ideas for Increasing Access to High-Quality Services
}

\author{
Emily Jordan Jensen, PhD, LMFT \\ Tai Mendenhall, PhD, LMFT \\ Catherine Futoransky, BS \\ Kirby Clark, MD
}

\begin{abstract}
Primary care settings often function as the front lines for behavioral health services in rural areas. The lack of formal behavioral health care in rural areas is also well documented. Rural family practice physicians were interviewed regarding the state of behavioral health care in their communities and their ideas for increasing access to quality care. Thirteen family practice physicians in rural locations participated in in-depth semi-structured interviews. Interviews were transcribed, coded, and analyzed following a phenomenological design. Physicians described a lack of quality behavioral health services and challenges for integrating and collaborating with those that do exist. Participants also described the changing role of stigma, service delivery strategies that are currently working, and the unique role primary care plays in rural behavioral health care. Several ideas for increasing access to and efficacy of services are discussed; these ideas are informative for future research and interventions.
\end{abstract}

Address correspondence to Emily Jordan Jensen, PhD, LMFT, Department of Family Social Science, University of Minnesota, 290 McNeal Hall, 1985 Buford Ave., Saint Paul, MN 55108, USA. Email: jorda675@umn.edu.

Tai Mendenhall, PhD, LMFT, Department of Family Social Science, University of Minnesota, Saint Paul, MN, USA. Catherine Futoransky, BS, Department of Family Social Science, University of Minnesota, Saint Paul, MN, USA.

Kirby Clark, MD, Department of Family Social Science, University of Minnesota, Saint Paul, MN, USA.

Journal of Behavioral Health Services \& Research, 2021. 554-565. (C) 2021 National Council for Behavioral Health. DOI 10.1007/s11414-021-09752-6 


\section{Introduction}

Existing evidence indicates that there is a shortage of mental health professionals in rural areas. ${ }^{1,2}$ As of $2017,62 \%$ of designated mental health shortage areas were situated in rural contexts. $^{3}$ This is noteworthy because significant concerns relating to mental health rates, severity, and outcomes persist for rural communities. ${ }^{4,5}$ Compared to urban counterparts, rural respondents are more likely to describe their mental health status as poor. ${ }^{5}$ They are also more likely to report higher levels of depression, suicide, substance abuse, domestic violence, and child abuse. 6,7

A growing body of research has been investigating the overlap of behavioral health care in primary care settings and has found promising indications regarding the efficacy of care and increased accessibility. ${ }^{1,8-10}$ While rates of psychiatrists per capita rapidly decrease as levels of rurality increase, the rate of family medicine physicians providing mental health care significantly increases in rural settings. ${ }^{11}$ Similarly, Miller and associates ${ }^{12}$ indicated that as rurality increased, the prevalence of mental health care services being co-located within primary care settings also increased. Data also show that primary care physician availability is associated with better mental health ratings for rural communities. ${ }^{13}$

In a landmark study of hospitals that integrated mental health services in 22 US states, Bird and associates ${ }^{14}$ proposed a model of four ways in which behavioral health care was integrated within primary care: (a) diversification (mental health care provided directly onsite), (b) linkage (an independent mental health practitioner or agency operates onsite), (c) referral (formal or informal arrangements made for patients to see offsite mental health professionals), and (d) enhancement (training primary care physicians to recognize, diagnose, and treat behavioral health concerns independently). Examples of each of these models were present in the primary care settings represented in this investigation. Much research on integrated care and updated models has been proposed. ${ }^{15,16}$ Bird et al.'s ${ }^{14}$ model, however, was developed specifically for rural contexts.

There is a growing body of research regarding the presence and function of barriers to behavioral health care. ${ }^{2,4,17}$ Penchansky and Thomas ${ }^{18}$ proposed a model for conceptualizing barriers that outlines dimensions of access: (a) affordability, (b) accessibility (location), (c) availability (number of providers), (d) acceptability (attitudes), and (e) accommodation (the relationships between the way services are organized and patients' abilities to integrate said services into their schedules and lives). This model focuses more specifically on fit between patients and the health care system. ${ }^{18}$ These concepts have been revised and adapted in rural behavioral health research, and the factors have appeared as a consolidated list of three service-related foci: availability, accessibility, and acceptability. ${ }^{4,19,20}$ Further understandings about the presence and function of these barriers were explored in the investigation presented here.

\section{Research Questions}

The present study was positioned to address a knowledge gap in understanding about how barriers to behavioral health care function for rural populations. The two grand tour questions that guided this investigation were: (a) What experiences and observations of barriers to behavioral health care among patients and their families do rural physicians witness in their practices? and (b) What ideas do rural physicians have for overcoming barriers to behavioral health care?

By interviewing Family Medicine physicians, this investigation also elucidated existing strategies that are working and/or not working and contributed to ideas about what steps are next in terms of inquiry and intervention aimed at reducing barriers to rural behavioral health care. 


\section{Method}

\section{Research Design}

This investigation was informed by phenomenological and hermeneutic phenomenological research designs, which follow the study of experiential meanings as they are lived out and present themselves in human consciousness. ${ }^{21}$ These approaches contribute to the understanding of observed phenomenon via rich descriptions of the personal and professional experiences of those immersed in the said phenomenon as it is occurring (in this case, physicians' experiences in rural communities with barriers to behavioral health care). Hermeneutic phenomenology has been applied to studies of the experiences of rural physicians before ${ }^{22,23}$ and was chosen for this investigation because of its interpretive strengths and the way it centers the meaning-making and expertise of the study participants themselves. The research design distilled meaning about the essence of rural physicians' experiences from a vast number of words used by the participants as they told stories, shared opinions, and engaged in critical thought about their communities and patients' experiences. Hermeneutic analysis originated in philosophical literature, ${ }^{24}$ and when it is applied to research, data are converted into written documents (in this case transcribed interviews), which then become the subject of analysis.

Research design diverged from pure hermeneutic phenomenology in that the bracketing of researcher assumptions and biases was intentionally prioritized. In hermeneutic phenomenology, researchers and study participants are viewed as co-creators of meaning, and researchers' own experiences, ideas, and assumptions are intentionally imbedded into the analysis. ${ }^{24}$ Both traditions value reflexivity, the process of self-reflection, and identification of biases and assumptions, but in phenomenology, reflexivity is followed by bracketing, the intentional separation of the self from the interpretation of findings. ${ }^{24}$ In this study, the following strategies were used to enhance the process of reflexivity and the subsequent bracketing of the self. The first strategy was memoing. Notes about opinions, emerging ideas, hypotheses, and personal responses were kept throughout the data collection and analysis phases. These notes were discussed throughout the research process during weekly research meetings. Ongoing analytic memos were kept in the coding documents to mark specific instances where perceived subjectivity or lack of certainty could be influencing data analysis. These instances were also discussed in research meetings. The researchers also kept an ongoing paper trail to log research activity, decisions, and milestones throughout the entire research process.

The interviews were guided by semi-structured questions that were created through consulting existing research, human ecology theory, ${ }^{25,26}$ and the Andersen model of health service use. ${ }^{27,28}$ The human ecology assumption that "environments do not determine human behavior, but pose limitations and constraints as well as possibilities and opportunities"29 (p. 426) informed interview questions related to perspectives on barriers to care and ideas physicians had for increasing access to behavioral health care in their practices.

An initial interview schedule was revised after a practice interview was conducted with a rural family physician who practiced outside of the sampling region for this study. Interviews were conducted via telephone or online video-conferencing (depending on participants' preferences) and lasted approximately $30 \mathrm{~min}$. All were audio-recorded and transcribed verbatim. Transcripts were then reviewed and edited for accuracy.

\section{Sampling and Recruitment}

Potential participants were identified by creating a list of every zip code contained in a ninecounty area of the Upper Midwest. The Minnesota Department of Human Services (MDHS) Provider Directory ${ }^{30}$ and the Wisconsin Medical Society (WMS) Physician Directory ${ }^{31}$ were used 
to search for family medicine physicians in each respective zip code. The directories included physician names, public addresses, and phone numbers for general hospital systems and clinics. The search revealed a total of 172 family medicine physicians that practiced in the range of zip codes represented by the nine-county area. As the scope of this investigation was on the experiences of rural communities, 71 potential participants were initially removed from the list because they practiced in the zip codes that covered two large cities (as identified by Rural Urban Commuting Area (RUCA) Codes $^{32}$ ). RUCA Codes are a zip-code level measurement system that calculates level of rurality based on population density, urbanization, and daily commuting patterns

in and out of each zip code. The most current codes are based on 2010 US Census data. ${ }^{32}$ After removing the urban providers, 101 potential rural physicians were left to be recruited. The list of 101 potential physicians was initially reviewed by the director of the University of Minnesota Medical School's Rural Physician Apprenticeship Program (RPAP) for potentially willing participants based on his familiarity and past work experience with physicians on the list. The director's perusal resulted in a list of seven physicians who were then contacted in the first wave of recruitment. A recruitment script was created, and potential participants were cold-called.

After the first wave of recruits had been contacted, a second wave of potential participants was identified based on the level of rurality in which they practiced. Rurality was measured using RUCA Codes, ${ }^{32}$ and the intention was to prioritize recruiting to those who were experiencing the greatest degree of rural practice. Three waves of phone-based recruitment were identified using this method, yielding 11 participants. Another participant was recruited through a personal connection with the first author and one more by snowball sampling.

\section{Sample Demographics}

Thirteen physicians participated in the study. They ranged in years of post-residency experience from less than 2 years to 48 years in practice (mean $=20.27$ years). Most had been at their present locations for the majority of their careers, and all but one had been in rural settings for their entire careers. See Table 1 for demographic details about the participants.

\section{Analysis}

There is variation to the analytical methods used in hermeneutic phenomenology when applied to research, ${ }^{33}$ but three key strategies make up the hermeneutic cycle: reading, reflective writing, and interpretation. ${ }^{33}$ Inspired by these strategies, in this study, three waves of coding analysis-holistic, detailed, and interpretive-were employed. ${ }^{21,34}$ This sequence served to distill and extract meaning from, and identify themes within and across, the interviews. During the first wave, text was read through in its entirety and coded using selective highlighting to distinguish meaning-rich passages. At the completion of this wave, holistic summaries of each interview were created. During the second wave, interviews were read again, this time to synthesize and describe passages of meaningful text at an immediate level. During this wave, codes followed as closely to the language used by the participants as possible. The third wave of coding was interpretive in nature, whereby efforts to ascribe meaning to the distilled ideas and tie them to the emerging themes from the rest of the interviews were advanced.

The interpretive wave of coding is where the protocol diverged from a pure hermeneutic phenomenology procedure and instead followed a more objective approach akin to phenomenology. Bracketing strategies were employed throughout the analysis process, but in this interpretive stage, they were used with greatest emphasis. Ongoing analytic memos were recorded to reflect emerging ideas about potential themes and to note personal biases that could influence objectivity. After the first four interviews were analyzed, preliminary codes and recurring ideas were noted. After each subsequent interview was coded, revisions and additions were made to the list. The first 
Table 1

Demographic information for rural physician study participants

\begin{tabular}{lllll}
\hline ID number & RUCA code & Years in practice & Gender & Current location/specialty \\
\hline 1 & 4 & 21 & Female & ER \\
2 & 10.3 & 37 & Male & Family Medicine \\
3 & 10.3 & 48 & Male & ER \\
4 & 7 & 17 & Male & Family Medicine \\
5 & 10.3 & 7 & Female & Family Medicine \\
6 & 10.3 & 1.5 & Female & Family Medicine \\
7 & 10 & 3 & Male & Family Medicine \\
8 & 4.1 & 26 & Male & ER \\
9 & 4 & 32 & Female & Family Medicine \\
10 & 4 & 20 & Female & ER \\
11 & 4.1 & 5 & Female & Family Medicine \\
12 & 7.2 & 26 & Male & Family Medicine \\
13 & 10.3 & 20 & Female & Family Medicine \\
\hline
\end{tabular}

author coded all 13 interviews, the second author coded six interviews, and the third author coded three-all following this exact coding protocol. Reconciliation meetings among the researchers were conducted throughout the process and were used to identify differences in interpretations, bracket assumptions and biases, refine the coding protocol, and discuss emerging themes. At the end of the coding process, the list of emerging themes, categories, and subcategories was reorganized, revised, and run through peer-checks (with fellow researchers) and member-checks (with interviewees) as another measure to ensure that data interpretations were trustworthy and representative and that individual assumptions had been bracketed and set aside.

\section{Results}

Findings were synthesized into seven overarching themes, each with categories and subcategories. What follows here are reported data and descriptions of each theme.

\section{Family Physicians Are Intentional in Their Choices to Practice in Rural Communities}

Participants were asked to share their reasons for choosing rural practice; all reflected a sense of intention. Most had been in their current practice locations for the majority of their careers. For those that had changed locations, all but one had been in rural areas for the entirety of their career. Reasons for this ranged from personal (e.g., enjoyed proximity to nature, grew up in rural areas, had family in rural communities, liked small towns) to professional (e.g., family medicine is suited to rural practice, family medicine allows an extensive range of medical practice, loan repayment programs). These findings were consistent with existing research about characteristics of rural physicians $^{35}$ but were also unique in the way the personal reasons were emphasized by the physicians in this study.

Existing research has found that compared to urban counterparts, rural physicians are similarly compensated but tend to work longer hours and see more patients. ${ }^{36}$ For the physicians in this study, more workload seemed to be offset by a strong sense of liking the work and the 
communities, especially among those who had lived there for many years. Speaking to the culture of their small community, one physician stated, "So, the thing that some people find creepy is that you go to the grocery store and everyone knows who you are. I find it fascinating. Comforting." These reflections about rural communities often segued into ideas about the culture of the communities themselves.

\section{Rural Culture Presents Challenges and Opportunities for Behavioral Health}

Respondents described several unique features about their communities. One that came up frequently had to do with the tight-knit, interconnected nature of rurality, e.g., "I always joked that you had to be nice to everyone because something will break in your house and your friend's exbrother-in-law will be the only one who could fix it." Comments about everyone knowing everyone else were commonplace. One shared this example of lack of anonymity impeding care with a behavioral health provider:

It's even come up where the counselors felt uncomfortable with some things. There was one episode where she was counseling a parent at a child visit, well it turns out that the counselor's kid had bit her kid the day prior at daycare.

So, there is just a lot of that.

Physicians also discussed the implications of lack of anonymity on health care and shared that they often treat entire families within their practices

Several physicians mentioned job loss and economic insecurity as causes for family instability and behavioral health concerns. Poverty was frequently described both as a cause for behavioral health and health concerns and as a barrier for seeking and receiving appropriate treatment. Another unique feature of rural communities that was described as a barrier to receiving care was a general fear or mistrust of cities and of outsiders. One participant described this as "tribalism" and went on to say:

We have a lot of people that are fearful of going through to the big city. We have a lot of people who have never traveled on an airplane or ... met a lot of people outside of their own racial group or their own socioeconomic group.

These and other cultural factors (including how the presence of American Indian reservations and historic immigrant communities shape culture, the importance of industry to rural towns, and the effects of remote location of these communities) were described by participants in the beginning of interviews and referenced back frequently as they discussed implications for behavioral health.

\section{A Range of Behavioral Health Concerns Persist for Rural Communities}

Physicians discussed several behavioral health concerns that persist for their patients and several shared ideas about factors that cause and/or exacerbate presenting symptoms. All described anxiety and depression among their patients, and many discussed substance abuse (alcohol, opioids, etc.) as a co-occurring and/or separate concern. One physician shared, "The opioid epidemic is alive and well. A lot of heroin abuse. A lot of prescription drug abuse. A lot of deaths related to that."

Deaths by suicide and emergency behavioral health situations were described by several physicians. One explained that behavioral health concerns and suicide rates may be inflated not by residents of the community but by individuals who travel to the area as a destination for escaping perceived problems and carrying out suicide plans: "The other thing that I was not aware of, and has been an unfortunate reality of being here is, I forget the exact term they've dubbed it, but 'suicide tourism', where people will come here and commit suicide."

Other behavioral health concerns were mentioned with lower frequency but often reflected concerns with specific age groups. Age-related concerns for elderly patients, such as dementia, 
were mentioned, as were concerns over the dearth of care available for children's behavioral health services.

Physicians often thought holistically about patients' circumstances and offered hypotheses about the causes for behavioral health concerns in their communities. Ideas included aging, family instability and stress, historic trauma and oppression, social isolation, unemployment, and poverty. With those who treat younger patients, stressors related to social media were mentioned and were also described.

\section{Existing Services Are Often Insufficient or Disconnected from Health Care}

Physicians described a range of existing behavioral health resources, including medication management (handled by nurse practitioners, family practice doctors, and-in rare cases - psychiatrists), emergency room services, and some private and community behavioral health services. In some cases, integrated social work and counseling, crisis response teams, and telehealth services for emergency assessment (and in a minority of cases, ongoing telehealth therapy) were also present.

Several categories of concerns about existing services emerged across the interviews, exposing shared challenges with existing behavioral health services. These included limited availability of services, inconsistent availability of services, lack of access to quality services, and lack of collaboration among providers advancing said services.

In sum, most participants were concerned about the availability of behavioral health services. In rare cases, some expressed that behavioral health resources had improved over time in their communities. More frequently, however, they expressed concern about services having left their communities, and that there were several limitations with the services that did exist.

\section{Several Persisting Barriers Prevent Patients from Accessing Care}

Although all physicians identified resources that are available in their communities, they also all named barriers that prevent patients from accessing said resources. Several barriers are related to availability of providers (as described above), alongside issues with cost and lack of insurance, distance, patients not wanting to take the time to go to appointments, and symptoms from behavioral health concerns inhibiting patients' abilities to attend appointments.

Other, more frequently mentioned, barriers had to do with patients' attitudes towards and perceptions of behavioral health concerns and care. These included a lack of trust for providers, lack of anonymity around help-seeking, and behavioral health stigma. One unique finding was that many physicians, especially those who had been in practice for many years, described observing a decrease in the effects and presence of behavioral health stigma for their patients. Some explained that it was still present for older patients but that (likely due to media exposure) it has been reducing with younger generations and in general.

\section{Family Physicians Have Unique Roles in Rural Practice}

As described by one participant, family physicians are the "front lines" for all health care in rural communities (including behavioral health care). This position means that family physicians often take on unique roles and duties. Some participants described doing assessment and triage services for behavioral health concerns. These physicians moved from the "front lines" role to more of a bridging role, connecting patients to more specified services if and as they were available. Some physicians also described doing basic behavioral therapy interventions and attempting to destigmatize mental illness and associated services. Concerns around medication management were mentioned several times, as well. Some physicians explained that they have to practice at the very 
edge of their competence when it comes to psychiatric medication. Many maintained that increased training, providers, and resources would ease some of the difficulties and pressures they experience. Several explained that their unique roles, too, result in awareness of family dynamics and stressors can impact patients' behavioral health and behavioral health care.

\section{Rural Family Physicians' Unique Ideas for Increasing Access to Behavioral Health Care}

All participants offered ideas regarding how to increase behavioral health care in rural communities. Some of the ideas already being employed involved strategies for increasing the reach of existing behavioral health professionals. Some communities approach this by sharing social workers and counselors between clinics. Because small clinics cannot afford fulltime professionals on their own, they collaborate with other small clinics - and are able to meet needs that way. To increase the reach of psychiatrists, one clinic has monthly video consultations wherein all family physicians bring cases to discuss medications, diagnoses, and treatment plans. Two other physicians mentioned that they have regular phone access with a regional psychiatrist for similar assistance. Another creative strategy was employed by a small hospital when they added a behavioral health professional to their team:

When we integrated a behavioral health worker in our clinic, there was a concern that you can't just walk in the waiting room and say, you know, "the therapist will see you now." So we had to create an anonymous way to call people back like they were just regular clinic patients so they could be comfortable. We are just trying to be cautious about that because it is an issue in a small town.

Like the strategies that increase the reach of professionals, this does not cost extra money or take more time. These strategies are aimed at increasing the effectiveness of existing resources.

Ideas shared by participants for future strategies included a range of methods for building on existing resources and introducing new ones. Several spoke to the efficacy of telehealth technology. Others described a need for increased financial resources. One physician explained that "money talks" and that if reimbursement rates for psychiatry and behavioral health increased, access to quality care would too. Other ideas included better provider training for behavioral health concerns, rural training programs for behavioral health professionals, increases in collaboration with existing resources, culturally appropriate treatment, increasing the number of professionals who are able to prescribe medications, programs to aid with transportation to appointments, and prevention-oriented school programs. One commonly mentioned idea was increasing community awareness of behavioral health concerns and services through education and outreach.

\section{Implications for Behavioral Health Services}

The themes described above represent the essence of these rural physicians' experiences. In following the hermeneutic phenomenological approach, the interpretations have been guided by the language of the physicians themselves - centering their lived experiences and personal meaningmaking. Several of the findings that emerged from this process are noteworthy. One key finding that largely confirmed existing research is that behavioral health care is integrated within primary care in these rural communities (to varied degrees and through varied means). All respondents described behavioral health services and resources that are available to patients, and, alongside these descriptions, several strengths and weaknesses of available services emerged. The four methods of behavioral health integration described by Bird et al. ${ }^{14}$-including diversification, linkage, referral, and enhancement-were all put forth as models that these communities are using or have used in the past. For each, specific associated barriers to the method of integration were also identified. For diversification (integrating behavioral health professionals directly onsite), lack of funding, lack of providers, inconsistency, inability to meet demand, and lack of trust for 
providers were all listed as barriers. For linkage (integrating independent, outside professionals and agencies onsite), barriers related to funding, lack of providers, and inconsistency were mentioned. As an opportunity, some clinics integrated outside agencies via telehealth technology. For the model of referral (formal and informal arrangements for care with outside professionals), lack of availability of providers, lack of quality providers, stigma, distance, cost, lack of communication and collaboration with health care, and lack of awareness of existing community resources were all mentioned. For the model of enhancement (training primary care professionals to identify, diagnose, and treat behavioral health concerns), the only barrier mentioned was a lack of training. Several identified this integration model as a future idea for increasing access to care.

Referral to outside behavioral health sources may be the most apparent integration method, but it was also the method associated with the most barriers based on the physicians' reports. Lack of provider availability in rural areas and challenges for existing rural behavioral health providers have been well documented. , $^{37,37,38}$ These findings, combined with evidence from existing literature, suggest that more effective strategies for increasing access to behavioral health care in rural communities may involve integrating and maximizing existing resources instead of creating space for and recruiting new services to these areas.

Another key finding is that the presence and impact of behavioral health stigma has been decreasing according to physicians in the present investigation. This finding is in line with some research, ${ }^{39}$ but challenges more established ideas regarding stigma as a barrier to behavioral health care. $^{7,40-42}$ Though it was still mentioned as a barrier by physicians, the main takeaway in this study is that its presence and impact have diminished over time. Physicians hypothesized that media has played a large role in normalizing behavioral health concerns and services; if this trend continues, stigma may be a smaller barrier to overcome as time goes on. Despite this, however, the presence of behavioral health stigma was still mentioned as a barrier by nearly every physician. If physical health care carries less stigma than behavioral health care (and it does), then increased integration of behavioral health care into primary care settings continues to be indicated. ${ }^{43,44}$ As primary care physicians, the respondents in this study may have experienced less behavioral health stigma in their practices because they themselves were actors in the efforts to reduce stigma. Primary care may not carry the same stigma that psychiatry or behavioral health treatment does, and for this reason, it may be a more welcoming entry point for those for whom stigma is a barrier to needed care.

\section{Strengths and Limitations}

Rural family physicians have participated in surveys and a mixed methods investigation about perspectives on behavioral health before, ${ }^{9}$ but this qualitative study is the first that used in-depth interviews about physicians' experiences with and perspectives regarding behavioral health care in rural communities. The range of opinions brought forth represents an important contribution to our understanding about behavioral health barriers. Practices of trustworthiness and reflexivity, too, represent strengths of this study in its informing of accurate findings. Attention to the geographic loci (using RUCA code measurements) also ensured that participants represented rural communities - versus small towns or colloquially "rural" areas.

Limitations of this study are important to consider, as well. First, recruitment methods relied on convenience and snowball sampling strategies in order to reach a meaningful sample of rural family physicians. Though high levels of saturation did occur across the 13 interviews, a larger sample could provide more insights. Second, self-selection may have shaped participants' responses. It is possible that physicians who already had an interest in behavioral health concerns were the most likely to agree to participate in the study. They thereby could have presented stronger narratives regarding health care services as amenable to behavioral health needs. 
Finally, while generalizability is not always primary goal of qualitative research, ${ }^{45}$ it should be noted that all responses came from a seven-county region in a North Central US State. As a location-based variable, rurality is difficult to operationalize and generalize across studies. These responses reflect rural culture for their specific region, but it should be noted that rural cultures (i.e., situational and contextual factors for each region) vary. Findings presented here should be interpreted with this understanding.

\section{Next Steps for Research and Intervention}

Future research should continue to explore the efficacy of strategies for integration of behavioral health services within primary care locations. It would be beneficial to interview and survey residents of rural communities who do not have professional or medical backgrounds as well. While it can be difficult to access patients as research participants, their voices and experiences will be invaluable in shaping appropriate and effective interventions. The respondents in this study have specific views as physicians, and it makes sense that integration with primary care services would be a key focus of their ideas for improving care.

Future investigations should also consider the possibility of integrating behavioral health services with other community institutions (e.g., first responders, churches, schools). There was a high level of focus in the present study on the dearth of emergency services, but not much attention to preventative behavioral health care. It is likely that as the front lines for behavioral health emergencies, exacerbated conditions, assessment, triage, and treatment were more salient in their responses. However, preventative approaches may lead to lower costs and better outcomes. ${ }^{46}$ It is likely that rural residents are interfacing with other community institutions more regularly and doing this long before they access health care systems. More research is needed to understand ways to access preventative care within existing social structures (e.g., school programs that advance stress-reduction and/or mindfulness-based interventions) and to evaluate its reach and effectiveness.

\section{Conclusion}

This investigation represents a unique contribution to existing literature about barriers to rural behavioral health care and regarding the integration of behavioral health care into primary care settings. Experiences from a range of family practice physicians revealed several challenges and opportunities. Key findings suggest that barriers to behavioral health care services are experienced differently depending on the type of services and level of integration with primary care that is available. More research is needed to advance strategies for integrating already-extant behavioral health resources into both rural health care systems and other community institutions.

\section{Declarations}

Conflict of Interest The authors declare no competing interests.

\section{References}

1. Mohatt DF, Bradley MM, Adams SJ, et al. Mental health and Rural America: 1994-2005. Washington, DC: United States Department of Health and Human Services, Health Resources and Services Administration, Office of Rural Health Policy. 2006; https:// www.ruralhealthresearch.org/mirror/6/657/RuralMentalHealth.pdf. Accessed April 30, 2018. 
2. Thomas D, Macdowell M, Glasser M. Rural Mental Health Workforce Needs Assessment: A National Survey. Rural \& Remote Health. 2012;12(4):1-12. https:/www.rrh.org.au/journal/article/2176. Published October 8, 2012. Accessed February 1, 2019.

3. United States Department of Health and Human Services, Health Resources and Services Administration. Designated Health Professional Shortage Areas (HPSA) Statistics. Available online at https:/ersrs.hrsa.gov/ReportServer?/HGDW_Reports/BCD_HPSA/ BCD_HPSA_SCR50_Smry\&rs:Format=PDF. Published 2018. Accessed February 1, 2019.

4. Smalley K, Warren J. The current state of rural mental health. In K Smalley, J Warren, and J Rainer (eds.), Rural Mental Health: Issues, Policies, and Best Practices. New York, NY: Springer; 2012, pp. 3-16.

5. Ziller EC, Anderson NJ, Coburn AF. Access to Rural Mental Health Services: Service Use and Out-of-Pocket Costs. Journal of Rural Health. 2010;26:214-224.

6. Eberhardt MS, Pamuk ER. The Importance of Place of Residence: Examining Health in Rural and Nonrural Areas. American Journal of Public Health. 2004;94(10):1682-1686.

7. Smalley KB, Yancey CT, Warren JC, et al. Rural Mental Health and Psychological Treatment: A Review for Practitioners. Journal of Clinical Psychology. 2010;66(5):479-489.

8. Fitzpatrick SJ, Perkins D, Luland T, et al. The Effect of Context in Rural Mental Health Care: Understanding Integrated Services in a Small Town. Health \& Place. 2017;45:70-76.

9. Taylor R, Glasser M, Hinkle H, et al. Meeting the Mental Health Needs of Rural Veterans Through Primary Care Providers. Journal of Rural Mental Health. 2016;40(2):124-135.

10. Williams D, Eckstrom J, Avery M, et al. Perspectives of Behavioral Health Clinicians in a Rural Integrated Primary Care/Mental Health Program. The Journal of Rural Health. 2015;31(4):346-353.

11. Xierali IM, Tong ST, Petterson SM, et al. Family Physicians are Essential for Mental Health Care Delivery. The Journal of the American Board of Family Medicine. 2013;26(2):114-115.

12. Miller BF, Petterson S, Levey SMB, et al. Primary Care, Behavioral Health, Provider Colocation, and Rurality. Journal of the American Board of Family Medicine. 2014;27(3):367-374.

13. Peterson LE, Tsai AC, Petterson S, et al. Rural-Urban Comparison of Contextual Associations with Self-Reported Mental Health Status. Health \& Place. 2009;15(1):125-132.

14. Bird DC, Lambert D, Hartley D, et al. Rural Models for Integrating Primary Care and Mental Health Services. Administration and Policy in Mental Health. 1998;25(3):287-308.

15. Heath B, Wise Romero P, Reynolds KA. Review and Proposed Standard Framework for Levels of Integrated Healthcare. Available online at integration.samhsa.gov.https://www.integration.samhsa.gov/integrated-care-models/ A_Standard_Framework_for_Levels_of_Integrated_Healthcare.pdf. Published March, 2013. Accessed March $30,2019$.

16. Peek CJ. Integrated Care: Aids to Navigation. Presented at Eastern Ohio \& West Virginia Summit. Pittsburgh, PA, 2007.

17. Jensen EJ, Wieling E, Mendenhall T. A Phenomenological Study of Clinicians' Perspectives on Barriers to Rural Mental Health Care. Journal of Rural Mental Health. 2020;44(1):51-61.

18. Penchansky R, Thomas JW. The Concept of Access. Medical Care. 1981;19(2):127-140.

19. Human J, Wasem C. Rural Mental Health in America. American Psychologist. 1991;46:232-239.

20. Jensen EJ, Mendenhall T. Rural Mental Health Research in Family Science. Journal of Contemporary Family Therapy. 2018;40(4):309-317.

21. Van Manen M. Researching Lived Experience: Human Science for an Action Sensitive Pedagogy. Albany, NY: State University of New York Press; 1990.

22. Konkin J, Grave L, Cockburn E, et al. Exploration of Rural Physicians' Lived Experience of Practising Outside Their Usual Scope of Practice to Provide Access to Essential Medical Care (Clinical Courage): An International Phenomenological Study. BMJ Open. 2020;10(8).

23. Snadden D, Reay T, Hanlon N, Macleod M. Engaging Primary Care Physicians in System Change - An Interpretive Qualitative Study in a Remote and Rural Health Region in Northern British Columbia, Canada. BMJ Open. 2019;9(5).

24. Laverty SM. Hermeneutic Phenomenology and Phenomenology: A Comparison of Historical and Methodological Considerations. International Journal of Qualitative Methods. 2003;2(3):21-35.

25. Bronfenbrenner U. The Ecology of Human Development: Experiments in Nature And Design. Cambridge, MA: Harvard University Press; 1979.

26. Keefe D. Transactions: The butterfly model. In D. Keefe (ed). Time in Human Ecological Conceptual Systems: Transactions, Transformations, and Tension. East Lansing, MI: Michigan State University Libraries; 2015, pp. 21-48.

27. Andersen RM. Revisiting the Behavioral Model and Access to Medical Care: Does it Matter? Journal of Health and Social Behavior. 1995;36:1-10.

28. Andersen R, Davidson P, Baumeister S. Improving access to care. In G. F. Kominski (ed). Changing the U.S. Health Care System: Key Issues in Health Services Policy and Management. San Francisco, CA: Jossey-Bass; 2014, pp. 32-62.

29. Bubolz M, Sontag M. Human ecology theory. In P. G. Boss, W. J. Doherty, R. LaRossa, et al. (eds). Sourcebook of Family Theories and Methods: A Conceptual Approach. New York, NY: Plenum Press; 1993:419-448.

30. Minnesota Department of Human Services. Provider search. http://mhcpproviderdirectory.dhs.state.mn.us/. Accessed September 1, 2018.

31. Wisconsin Medical Society. Physician Directory. Available online at https:/www.wisconsinmedicalsociety.org/resources/physiciandirectory/. Accessed September 1, 2018.

32. United States Department of Agriculture, Economic Research Service. Rural-Urban Commuting Area Codes. Available online at https://www.ers.usda.gov/data-products/rural-urban-commuting-area-codes.aspx. Updated July 3, 2019. Accessed August 1, 2018.

33. Kafle NP. Hermeneutic Phenomenological Research Method Simplified. Bodhi: An Interdisciplinary Journal. 2013;5(1):181-200.

34. Van Manen M. Phenomenology of Practice: Meaning-Giving Methods in Phenomenological Research and Writing. London, England: Routledge, Taylor \& Francis Group; 2014. 
35. Goodfellow A, Ulloa JG, Dowling PT, et al. Predictors of Primary Care Physician Practice Location in Underserved Urban or Rural Areas in the United States. Academic Medicine. 2016;91(9):1313-1321.

36. Weeks WB, Wallace AE. Rural-Urban Differences in Primary Care Physicians' Practice Patterns, Characteristics, and Incomes. The Journal of Rural Health. 2008;24(2):161-170.

37. Gonyea JLJ, Wright DW, Earl-Kulkosky T. Navigating Dual Relationships in Rural Communities. Journal of Marital and Family Therapy. 2014;40(1):125-136.

38. Domino ME, Lin C-CC, Morrissey JP, et al. Training Psychologists for Rural Practice: Exploring Opportunities and Constraints. The Journal of Rural Health. 2018;35(1):35-41.

39. Polaha J, Williams SL, Heflinger CA, et al. The Perceived Stigma of Mental Health Services Among Rural Parents of Children with Psychosocial Concerns. Journal of Pediatric Psychology. 2015;40(10):1095-1104.

40. Ahmedani BK. Mental Health Stigma: Society, Individuals, and the Profession. Journal of Social Work Values and Ethics. 2011;8(2):410 .

41. Larson JE, Corrigan P. The Stigma of Families With Mental Illness. Academic Psychiatry. 2008;32(2):87-91.

42. Larson JE, Corrigan PW, Cothran TP. The impact of mental health stigma on clients from rural settings. In KB Smalley, JC Warren, JP Rainer (eds). Rural Mental Health: Issues, Policies, and Best Practices. New York, NY: Springer; 2012, pp. 49-64.

43. Mukolo A, Heflinger CA. Factors Associated with Attributions About Child Health Conditions and Social Distance Preference. Community Mental Health Journal. 2010;47(3):286-299.

44. Kirchner J, Cody M, Thrush C, et al. Identifying Factors Critical to Implementation of Integrated Mental Health Services in Rural VA Community-Based Outpatient Clinics. Journal of Behavioral Health Sciences \& Research. 2004;31(1):13-25.

45. Carminati L. Generalizability in Qualitative Research: A Tale of Two Traditions. Qualitative Health Research. 2018;28(13):2094-2101.

46. Rishel CW. Evidence-Based Prevention Practice in Mental Health: What is it and How do We Get There? American Journal of Orthopsychiatry. 2007;77(1):153-164.

Publisher's Note Springer Nature remains neutral with regard to jurisdictional claims in published maps and institutional affiliations. 